\title{
Comparison of outcomes between odontogenic and non-odontogenic sinusitis after management of orbital abscess : a retrospective cohort study of 29 adults.
}

\author{
Maxime Salfrant ${ }^{1}$, Mathieu Veyrat ${ }^{1}$, Domitille Camous $^{1}$, Elias Maalab ${ }^{1}$, Franklin Lizé $^{1}$, \\ Romain Kania ${ }^{1}$, Nicolas LE CLERC ${ }^{1}$, Jean-Philippe Blancal ${ }^{1}$, Jean Pierre GUICHARD ${ }^{1}$, \\ Benjamin Verillaud ${ }^{1}$, and philippe herman ${ }^{1}$ \\ ${ }^{1}$ Groupe Hôpital Saint-Louis Lariboisière et Fernand-Widal
}

April 27, 2020

Keypoints and Key words

Keypoints :

- Many reports suggest that odontogenic sinusitis cause critical orbital abscess with high risk of ophthalmologic sequelae due to the pathogenic potential of anaerobic bacteria.

- This study aimed to compare the outcomes between odontogenic sinusitis and non-odontogenic sinusitis after management of orbital abscess in an adult population.

- The rates of revision surgery and visual sequelae were significantly higher in the odontogenic sinusitis group.

- Dental etiology of the sinusitis was overlooked in four patients (24\%) of the odontogenic sinusitis group. Hence, dental cause of sinusitis has to be systematically sought and treated.

Key words: Abscess, Blindness, Odontogenic infection, Orbital cellulitis, Sinusitis.

Ethical considerations

The protocol was approved by the local ethics committee. All participants gave informed consent.

Main Text

INTRODUCTION

Orbital cellulitis is a rare acute infection of the orbit and its contents that occurs mostly in children. The main cause is sinusitis (1). It is usually classified according to Chandler's classification based on location and severity (2). Among orbital cellulitis, abscess formation occurs in $30 \%$ of the patients (3). Associated neurological acute complications, such as empyema or cerebral abscess, are rare but life-threatening. Long-term complications can be dramatic, notably blindness and cosmetic sequelae. The treatment includes hospitalization, intravenous antibiotics and surgery. The surgical procedure can combine endoscopic endonasal approach and external approach.

According to the scientific literature, only $10 \%$ of sinusitis are odontogenic (4). Some authors suggest that odontogenic sinusitis (OS) cause critical orbital abscess (5) with higher risk of ophthalmologic sequelae (6), due to the pathogenic potential of anaerobic bacteria.

This study aimed to compare the outcomes between OS and non-OS after management of orbital abscess in an adult population. 


\section{MATERIALS AND METHODS}

\section{Study design}

This is a retrospective cohort study from a tertiary referral otorhinolaryngology department. This department is the location of a centralized Ear-Nose-Throat (ENT) ward for adult patients. We reviewed all medical records of patients hospitalized and operated for an orbital abscess complicating a sinusitis from January 2001 until December 2017.

\section{Participants}

We included consecutive teenagers over 15 years old and adult patients with stage III and IV orbital cellulitis according to Chandler's classification. The cause of the infection had to be a paranasal bacterial sinusitis. Patients were excluded in case of dacryocystitis, fungal sinusitis or mucocele. Patients with thrombosis of the cavernous sinus were also excluded because there was no associated abscess in the orbit.

\section{Data sources and Variables}

Patient's characteristics, clinical features at presentation, bacteriological samples, imaging (contrast-enhanced CT-scan and/or MRI), surgical procedures (endoscopic and/or external approach) were recorded. The choice of the surgical approach was made by the surgeon on ward. Surgical revisions and their causes were particularly studied. Revision surgery was decided based on the synthesis of careful monitoring of the patients and computed tomography.

The patients were classified in two groups: OS group, and non-OS group. In OS group, there was a clinical and/or radiographic evidence of a dental infection in relation to the pathologic maxillary sinus (carious tooth, tooth with defective restoration, or periapical lesion).

Characteristics at admission, acute / long term complications and rate of revision surgery were analyzed and compared between the groups.

Statistical methods

Statistical analysis was performed using Wilcoxon-Mann Whitney test and Fischer's exact test. A p value inferior or equal to 0.05 was considered as significant.

\section{RESULTS}

\section{Patients}

29 patients were included in the study, $17(59 \%)$ in OS group and $12(41 \%)$ in non-OS group. The age of the patients $(\mathrm{p}=0,04)$ and the proportion of visual impairment at admission $(\mathrm{p}=0,03)$ were higher in OS group (Table 1). In OS group, all patients presented at least a maxillary sinusitis. In non-OS group, ethmoiditis was the commonest cause of the infection. The rate of acute complications was not significantly different between the groups (Table 2). All patients were hospitalized, underwent biological examination and a contrast-enhanced CT-scan. They received broad-spectrum intravenous antibiotics: generally a bitherapy composed of a third generation cephalosporin and an imidazole. All patients underwent an ophthalmological evaluation. In OS group, bacteriological samples found mostly a mixed flora composed of anaerobes $(45 \%)$ and oral streptococci $(42 \%)$. While in non-OS group, oral streptococci were preponderant (50\%). No patient died because of orbital abscess or associated complications.

\section{Surgical approach}

A surgical drainage was systematically performed at patients' admission. 10 (34.5\%) patients underwent an external approach, $10(34.5 \%)$ an endoscopic approach, and $9(31 \%)$ a combined approach. The repartition of surgical approaches was not significantly different between the groups. The most frequent endoscopic endonasal procedures were middle meatal antrostomy (95\%) and anterior ethmoidectomy (84\%). Endoscopic orbital decompression was performed in five patients. 


\section{Revision Surgery}

The rate of revision surgery was significantly higher in OS group $(p=0,03)$ (Table 3$)$. The main cause of revision surgery in OS group was the persistence or the re-accumulation of intra-orbital abscess. Lateral canthotomy was mandatory for one patient of the OS group during revision surgery because of the development of an irreducible exophthalmos associated with vision loss. One patient of the OS group required two revision surgeries because of the re-accumulation of an intra-orbital abscess.

\section{Dental infection}

In OS group, the first molar was mostly involved (48\% of the patients) and apical granuloma was the commonest etiology for OS (82\%). Teeth extraction was performed in 7 patients of the OS group during the first surgery. During revision surgery, it was accomplished in 3 patients. The patient requiring 2 revision surgeries underwent tooth extraction during the second revision surgery. Dental treatment was conducted secondarily in two patients. In the four other patients, i.e. $24 \%$ of OS group, dental etiology of the sinusitis was overlooked until this retrospective study and a careful reviewing of CT-scans.

\section{Sequelae}

The rate of visual sequelae was higher in OS group $(\mathrm{p}=0,04)$ (Table 4$): 6$ patients suffered blindness and 2 patients presented a decreased visual acuity in OS group whereas one patient presented a decreased visual acuity in the non-OS group. Cosmetic sequelae were rare even though two patients required a reconstructive surgery : a lipofilling of the cheek and a Mustardé flap for the reconstruction of the lower eyelid. Long term frontal mucoceles occurred in 2 patients.

\section{DISCUSSION}

\section{Key findings}

In this study, a higher risk of revision surgery and visual sequelae was observed in patients with orbital abscess complicating OS compared to non-OS.

Strengths of the study

To our knowledge, this is the first study comparing the rate of revision surgery among orbital abscess between OS and non-OS. This study depicts a large series of orbital abscess complicating acute sinusitis with a dramatic outcome in OS group in spite of an aggressive management combining surgery and intravenous antibiotherapy. This confirms the more aggressive pattern of odontogenic orbital abscess.

\section{Comparisons with other studies}

In this study, $59 \%$ of the patients presented a sinusitis of dental origin while in the scientific literature $5 \%$ of orbital cellulitis are odontogenic (6). Thus OS might be a risk factor for abscess formation in orbital cellulitis. Patients' age was higher in OS group, which is consistent with the literature: OS usually arise in the fourth and fifth decades (7). As described in this study, OS are polymicrobial infections with mixed aerobic and anaerobic organisms. The most commonly involved teeth are molars.

Dental etiology of the sinusitis was determined retrospectively in 4 patients, i.e. $24 \%$, of OS group. Particularly for OS, the clinical presentation is not always obvious as dental pain is often absent and less than half of patients report recent dental procedure (8). Furthermore, dental disease is often overlooked on CT scans leading to misdiagnosis. Searching and treating the odontogenic cause of the sinusitis is a key element in the management of these infections: concomitant treatment of the dental origin and the associated sinusitis will ensure resolution of the infection, and prevent recurrences and complications (4).

In this study, surgical management was characterized by its heterogeneity. Three different surgical approaches (external, endoscopic and combined) were performed; the procedures differed between each patient because of the variety of surgeons involved in the ward. In the literature, endoscopic sinus surgery tends to be the reference for orbital abscess complicating sinusitis (9). It is indeed considered as a safe, effective and minimally 
invasive procedure that allows to treat simultaneously the sinusitis and the orbital abscess. Nevertheless, some abscess can require an external approach after endoscopic sinus surgery, notably in case of lateral/superior orbital abscess and preseptal abscess. Dewan et al. in 2011 (10) report a higher risk of abscess re-accumulation in case of exclusive external approach. Thus, although an external approach might be associated depending on the extent of the infection, we recommend to systematically perform an endoscopic approach to improve the drainage.

Blindness was the worst sequela. Different mechanisms can lead to blindness: optic neuritis, ischemia resulting from thrombophlebitis of orbital veins, and orbital compartment syndrome. Orbital decompression has to be performed in extreme urgency in case of orbital compartment syndrome. In this study, the rate of visual sequelae was significantly higher for the OS group. This result reflects the findings of numerous authors reporting blindness as a frequent sequela of odontogenic orbital cellulitis (5). In a review of the literature, Youssef et al. in 2008 (6) noted that $46 \%$ of the patients developed blindness after odontogenic orbital cellulitis while only $10 \%$ of patients with orbital cellulitis complicating sinusitis suffer this complication (1).

Clinical applicability of the study

Orbital abscess complicating OS are severe infections with higher risk of revision surgery and visual sequelae compared to non-OS. Dental cause of sinusitis has to be systematically sought. An aggressive treatment is required with intravenous antibiotics and surgery. The surgery must drain the abscess, treat the sinusitis and its dental etiology.

References

1. Tsirouki T, Dastiridou AI, Ibánez Flores N, Cerpa JC, Moschos MM, Brazitikos P, et al. Orbital cellulitis. Surv Ophthalmol. 2018 Aug;63(4):534-53.

2. Chandler JR, Langenbrunner DJ, Stevens ER. The pathogenesis of orbital complications in acute sinusitis. The Laryngoscope. 1970 Sep;80(9):1414-28.

3. Van der Veer EG, van der Poel NA, de Win MML, Kloos RJ, Saeed P, Mourits MP. True abscess formation is rare in bacterial orbital cellulitis; consequences for treatment. Am J Otolaryngol. 2017 Apr;38(2):130-4.

4. Brook I. Sinusitis of odontogenic origin. Otolaryngol-Head Neck Surg Off J Am Acad Otolaryngol-Head Neck Surg. 2006 Sep;135(3):349-55.

5. DeCroos FC, Liao JC, Ramey NA, Li I. Management of odontogenic orbital cellulitis. J Med Life. 2011 Aug 15;4(3):314-7.

6. Youssef OH, Stefanyszyn MA, Bilyk JR. Odontogenic orbital cellulitis. Ophthal Plast Reconstr Surg. 2008 Feb;24(1):29-35.

7. Arias-Irimia O, Barona-Dorado C, Santos-Marino JA, Martínez-Rodriguez N, Martínez-González JM. Meta-analysis of the etiology of odontogenic maxillary sinusitis. Med Oral Patol Oral Cirugia Bucal. 2010 Jan 1;15(1):e70-73.

8. Little RE, Long CM, Loehrl TA, Poetker DM. Odontogenic sinusitis: A review of the current literature. Laryngoscope Investig Otolaryngol. 2018 Apr;3(2):110-4.

9. Teinzer F, Stammberger H, Tomazic PV. Transnasal endoscopic treatment of orbital complications of acute sinusitis: the Graz concept. Ann Otol Rhinol Laryngol. 2015 May;124(5):368-73.

10. Dewan MA, Meyer DR, Wladis EJ. Orbital cellulitis with subperiosteal abscess: demographics and management outcomes. Ophthal Plast Reconstr Surg. 2011 Oct;27(5):330-2.

\section{Hosted file}

Tables - Clinical Otolaryngology.docx available at https://authorea.com/users/315574/articles/

445923-comparison-of-outcomes-between-odontogenic-and-non-odontogenic-sinusitis-after-management- 
of-orbital-abscess-a-retrospective-cohort-study-of-29-adults 